\title{
LOS VALORES INTERPERSONALES Y SU RELACIÓN CON LOS ESTILOS ATRIBUTIVOS EN ESTUDIANTES DE QUINTO DE SECUNDARIA
}

\author{
INTERPERSONAL VALUES AND THEIR RELATION TO ATTRIBUTIONAL STYLES \\ IN FIFTH HIGH SCHOOL STUDENTS
}

Rosa Tueros C., María Matalinares, C. y Ornella Raymundo V.

UNMSM, LIMA - PERÚ

(ReCibIDo 13/02/13 AcEPTAdo 15/05/13)

\begin{abstract}
RESUMEN
La presente investigación tuvo como objetivo establecer la relación entre los valores Interpersonales y los estilos atributivos de estudiantes del $5 .^{\text {to }}$ año de educación secundaria. Se utilizó un método descriptivo correlacional, la muestra estuvo conformada por 400 alumnos, distribuidos proporcionalmente a la población. La investigación se llevó a cabo, utilizando el Cuestionario de estilos atributivos y el Cuestionario de Valores Interpersonales SIV de Leonard Gordon 1977. Los resultados indican de acuerdo con el coeficiente de correlación de Spearman que existe correlación significativa $(\mathrm{p}<0,05)$ entre los distintos valores interpersonales Soporte, Conformidad, Reconocimiento e Independencia con los Estilos Atributivos en el Área de Logros Académicos y Relaciones Interpersonales. Por el contrario no se obtuvieron correlaciones significativas entre los Valores Interpersonales Benevolencia y Liderazgo con los Estilos Atributivos en las Áreas de Logros Académicos y Relaciones Interpersonales.
\end{abstract}

Palabras clave: estilos atributivos, valores interpersonales, estudiantes de quinto grado de secundaria

\begin{abstract}
This research aimed to establish the degree of relationship between interpersonal values and attributional styles of male and female students of the $5^{\text {th }}$ year of secondary education. We used a descriptive correlational method, the sample consisted of 400 students, distributed in proportion to the population. The research was carried out, using the attributional style questionnaire and the Interpersonal Values Questionnaire SIV Leonard Gordon 1977. The results indicate according to the Spearman correlation coefficient exists correlation significant $(\mathrm{p}<0.05)$ between the different values interpersonal Support, Conformity, Recognition and Independence with attributional styles in the area of Academic Achievement and Interpersonal Relations. Instead no significant correlations between Interpersonal Values Compassion and Leadership attributional styles in Areas of Academic Achievement and Interpersonal Relations.
\end{abstract}

Keywords: attributional styles, interpersonal values high school fifth grade students 


\section{INTRODUCCIÓN}

En primer lugar es importante señalar que los valores concuerdan con nuestras concepciones de la vida y del hombre; no existen en abstracto ni de manera absoluta, se encuentran ligados a la historia, las culturas, los grupos humanos; influyen en nuestra forma de pensar, en nuestros sentimientos y formas de comportarnos; suponen un compromiso real y profundo de la persona ante sí misma y ante el medio social donde vive. Existen dos grandes grupos de valores que se dan en la sociedad: los valores relacionados con el desarrollo de una moral ciudadana y los valores que permiten a los individuos construir sus patrones autonomía, ser solidarios y vivir comprometidos con su sociedad. La escuela es la institución educativa importante que proporciona los cimientos que preparan al ser humano para la vida, impartiendo no solo conocimientos sino desarrollando y profundizando las actitudes y valores que requiere el estudiante a nivel intelectual, interpersonal y moral.

Los valores pueden constituir un medio para determinar lo que los sujetos hacen y cómo lo hacen; muchas de sus decisiones inmediatas, así como sus planes a largo plazo están influidas, consciente o inconscientemente, por el sistema de valores que adopten en Gordon (2003) citado en Emiro, Soto y Martínez (2009). De acuerdo a Gordon (1977) los valores se pueden apreciar en dos niveles: valores personales y valores interpersonales. Los valores interpersonales implicarían para el sujeto las siguientes características (Gordon 1995; citado en Emiro y cols. 2009):

Soporte (S): ser tratado con comprensión, recibiendo apoyo por parte de los demás; ser tratado con amabilidad y consideración.

Conformidad (C): hacer lo que es socialmente correcto, siguiendo estrictamente las normas; hacer lo que es aceptado e idóneo, ser conformista.

Reconocimiento $(\mathrm{R})$ : ser bien visto y admirado, ser considerado como persona importante, llamar favorablemente la atención, conseguir el reconocimiento de los demás.

Independencia (I): tener el derecho a hacer lo que uno quiera ser, ser libre para decidir por sí mismo, ser capaz de actuar según el propio criterio.

Benevolencia (B): hacer cosas por los demás y compartirlas con ellos, ayudar a los poco afortunados, ser generoso.

Liderazgo (L): estar a cargo de otras personas teniendo autoridad sobre ellas, estar en un puesto de mando o poder.

En el extranjero investigadores como Alonso, Pereira y Soto (2003); Alcalde, Marchena y García (2006) han realizado una serie de estudios sobre los Valores. Herrera (2007) realizó una investigación sobre los valores de los adolescentes de un centro español de educación secundaria en el extranjero (Marruecos), cuyos resultados fueron contrastados con adolescentes de otros lugares. Herrera y Herrera (2010) realizaron una investigación sobre los valores de los adolescentes y las de sus padres una investigación de un centro español de educación secundaria. 
En el Perú se han realizado algunas investigaciones con los Valores Interpersonales. Aguilar (2002) realizó un estudio comparativo de los valores interpersonales en alumnos con alta y baja autoestima con estudiantes universitarios de los tres primeros ciclos de una Universidad. Los resultados mostraron que existen diferencias significativas en el estudio del nivel Soporte como valor interpersonal entre los estudiantes con alta y baja autoestima. Existen diferencias significativas estudio del Valor Conformidad. No existen diferencias significativas el valor reconocimiento. Existen diferencias significativas en el Valor Independencia. No existen diferencias significativas en el Valor Interpersonal de Benevolencia. Existen diferencias significativas en el Valor Liderazgo. Los alumnos de alta autoestima poseen puntajes altos en los valores: liderazgo y benevolencia. Los alumnos de baja autoestima tienden a presentar bajo nivel en los valores de: soporte, conformidad, reconocimiento e independencia. Guerrero (2005) estudio la relación entre los acontecimientos vitales críticos y los valores interpersonales en los alumnos del $5 .{ }^{\text {to }}$ de secundaria. Encontró que existe relación entre los valores interpersonales de Reconocimiento y los Sucesos Críticos Vitales. Existe una relación inversa entre el Valor Interpersonal independencia y los Sucesos Críticos Vitales. No existe relación significativa entre los valores Interpersonales de Conformidad y Benevolencia y los Sucesos Críticos Vitales. Murakami (2005) investigó los Valores Interpersonales en Alumnos de 5. ${ }^{\text {to }}$ año de Educación Secundaria. Carmona y León (2005) investigaron la relación entre la inteligencia intrapersonal y los valores interpersonales en estudiantes del primer ciclo de Psicología de una Universidad Privada; encontraron que la Inteligencia Intrapersonal y los Valores Interpersonales son variables independientes que no guardan relación entre ellas. Romero y Villegas (2006) realizaron una comparación de los valores interpersonales de los estudiantes de primer ciclo con los del décimo primer ciclo. Los resultados mostraron diferencias entre ellos por ejemplo los alumnos del primer ciclo tienen mayor predominio de la escala de Soporte, tienden a una mayor necesidad de Reconocimiento, en tanto que los del Onceavo, revelan menor necesidad de Reconocimiento. El valor de Independencia y autonomía predominan en el grupo del Onceavo ciclo, en tanto que los alumnos del Primer ciclo denotan una tendencia hacia una adecuada socialización. Matalinares et al. (2009) realizaron un estudio sobre el juicio moral y valores interpersonales en estudiantes de secundaria de las ciudades de Lima y Jauja. Los resultados mostraron que las variables Juicio Moral y Valores Interpersonales se encuentran asociadas. El juicio moral se relaciona con las dimensiones conformidad, reconocimiento y benevolencia. En las dimensiones de conformidad, reconocimiento y benevolencia de los estudiantes se muestra diferente en función del sexo. Las mujeres obtienen mejores resultados que los varones, no sucede lo mismo en el caso del juicio moral. Por último, se encontraron diferencias significativas entre el juicio moral y los valores en función del grado de instrucción.

Ahora bien, respecto de la variable estilos atribucionales Fritz Heider inicia el método para evaluar cómo la gente percibe el comportamiento de sí mismo y de las demás personas, se refiere a cómo la gente genera explicaciones causales. 
Sustenta que todo comportamiento está determinado por factores internos o externos. En la atribución externa, la causalidad se asigna a un factor, a un agente o a una fuerza exterior. Los factores externos se salen fuera de su control. También conocida como atribución circunstancial. En la atribución interna, la causalidad se asigna a un factor, a un agente o a una fuerza interior. Los factores internos caen dentro de su propio control. Conocido también como atribución disposicional. Heider brinda una explicación del comportamiento humano limitada, puesto que tiende a atribuir los éxitos de otros y nuestras propias fallas a factores externos. No se percibe el éxito de otros como un mérito propio y tampoco las percibimos como nuestras propias fallas. Se tiende a atribuir nuestros propios éxitos y señalar como resultados de sus factores internos las fallas de los otros. Se percibe los éxitos propios como mérito personal y las fallas de los demás como sus propias responsabilidades.

Una de las teorías que se ha propuesto explicar la motivación humana en general, $\mathrm{y}$, en particular la motivación con que los alumnos afrontan el aprendizaje y el trabajo escolar ha sido la Teoría de la Atribución Causal de Weiner $(1979,1986$; en Tapia, Mateos, y Gutiérrez, 1994). Esta teoría parte de dos supuestos: primero, que las personas necesitan comprender y dominar el ambiente que les rodea y su propia vida para hacer que ambos sean algo predecibles y controlables; y, establece que se tratan de comprender, en particular, los determinantes causales de la propia conducta y de la de los demás (Gonzáles, 2005). Cabe señalar, que la teoría de la atribución causal tiene su origen en la teoría del sentido común de Heider y, en la actualidad, los aportes de Weiner han determinado que cada una de las causas poseen dimensiones causales que condicionarán las consecuencias psicológicas (cognitivas y afectivas) y el propio rendimiento final obtenido (Gonzáles, 2005). En este sentido, la conducta se considera como un continuo de episodios dependientes unos de otros, el éxito y el fracaso dan lugar, respectivamente, a respuestas emocionales positivas y negativas. Pero si los resultados son inesperados (aprobar cuando no se tenía esperanza de ello), negativos (reprobar, tanto si se esperaba como si no) o de gran importancia (conseguir ingresar a la universidad), tendemos a preguntarnos por las causas que los han determinado y a buscar respuesta a tales preguntas (Wong y Weiner, 1981; como se cita en Pardo y Tapia, 1990). Por otra parte, las explicaciones que nos damos influyen en nuestras respuestas emocionales y en nuestras expectativas y, a través de ellas, en el interés y esfuerzo que ponemos en conseguir alcanzar nuestras metas. Pues bien, desde la perspectiva del diagnóstico psicológico y cuando el objetivo del mismo es posibilitar la toma de decisiones de intervención en relación con la modificación del comportamiento del sujeto, la evaluación de las atribuciones interesa en la medida en que estas pueden actuar como variables determinantes total o parcialmente de los problemas motivacionales, por su repercusión sobre las expectativas y las respuestas afectivas del sujeto y, en consecuencia, sobre su conducta. Sin embargo, de cara a la intervención psicológica no solo interesa conocer las atribuciones específicas que el sujeto realiza ante determinados hechos, sino si tales atribuciones se dan de forma más o menos consistente, a través de 
distintos momentos y situaciones. Esto es, lo que interesa conocer y evaluar son sus "estilos atributivos" Metalsky y Abramson (1981).

En el extranjero Valle et al. (1997) analizaron la relación entre atribuciones y aprendizaje escolar, en concreto, el efecto de las atribuciones causales internas y externas sobre la utilización de diferentes enfoques de aprendizaje. Trataron de comprobar la influencia del hecho de atribuir los resultados académicos a factores causales internos (capacidad y esfuerzo) y externos (suerte) en la utilización de un enfoque de aprendizaje profundo o superficial. González, Valle, Suárez y Fernández (1999) realizaron un estudio sobre un modelo integrador explicativo de las relaciones entre metas académicas, estrategias de aprendizaje y rendimiento académico, este estudió planteó un modelo causal entre tres variables motivacionales (tipo de atribuciones, autoconcepto académico y metas de aprendizaje), las estrategias de aprendizaje significativo y el rendimiento académico. Buey y Romero (2003) estudiaron las relaciones existentes entre motivación de logro, estilos atributivos y expectativas de control, a partir de la información obtenida en las escalas de diversos cuestionarios entre ellos el cuestionario de estilos atributivos, aplicado a estudiantes de enseñanza Secundaria Obligatoria. Valenzuela (2007) en una investigación realizada en la ciudad de Merida, Chile, reporta evidencia empírica que apoyaría la idea de que una baja significancia en la exigencia académica en el plano escolar, tendría efectos negativos sobre la atribución que realizan los alumnos al esfuerzo, como rasgo explicativo del logro académico.

En el Perú son escasas las investigaciones sobre los estilos atribucionales, Tueros, Matalinares y Yaringaño (2009) realizaron la adaptación psicométrica del cuestionario de estilos atributivos. Matalinares et al. (2010) realizaron una investigación con la finalidad de estudiar la relación entre los estilos atribucionales y los estilos de aprendizaje en estudiantes de secundaria de Lima Metropolitana. Trabajaron con 364 sujetos de 14 a 17 años, de ambos sexos, de $4 .^{\circ}$ y $5 .^{\circ}$ grado de educación secundaria a quienes se aplicó el Cuestionario de Estilos Atribucionales propuesto por Alonso y Sánchez (1992) adaptado por Tueros, Matalinares y Yaringaño (2009) y el inventario de Estilos de Aprendizaje de Kolb (1979) adaptado por Escurra (1991). Los resultados mostraron que existe relación entre los estilos atribucionales y los estilos de aprendizaje. Se halló relación significativa entre las áreas que componen el aprendizaje experiencial y los estilos atribucionales. Existen diferencias entre los estilos atribucionales de varones y mujeres en las dimensiones de atribución del éxito académico a la habilidad y en la atribución del fracaso a la falta de habilidad. Los estilos de aprendizaje de varones y mujeres difieren en el área de experimentación activa. Por último, se halló que existe mayor preferencia de atribución al éxito en las relaciones interpersonales y una menor percepción de la atribución de fracaso al profesor. En el caso de los estilos atribucionales en el área de relaciones interpersonales, se presenta una preferencia por la Atribución del éxito en las relaciones interpersonales a la habilidad, así mismo se usa en menor medida la creencia sobre Externalización del fracaso. 


\section{HIPÓTESIS}

\section{Hipótesis general:}

Los valores Interpersonales se relacionan con los estilos atributivos en estudiantes del $5 .^{\text {to }}$ año de educación secundaria.

\section{Específicas:}

1. Existen diferencias en valores interpersonales de estudiantes del $5 .{ }^{\text {to }}$ grado de secundaria según la variable sexo.

2. Existen diferencias en estilos atributivos de estudiantes del $5 .{ }^{\text {to }}$ grado de secundaria según la variable sexo.

\section{MÉTODO}

En concordancia con la hipótesis formulada, se empleó el método descriptivo con un diseño transeccional correlacional y comparativo (Hernández, Fernández y Baptista 2006). La muestra de investigación estuvo conformada por 400 sujetos de ambos sexos, de $5 .^{\text {to }}$ grado de educación secundaria de instituciones estatales. Para la obtención de la muestra se aplicó un procedimiento de muestreo no probabilístico intencionado.

\section{Instrumentos}

1) El Cuestionario de Valores Interpersonales SIV propuesta por Leonard Gordon, fue traducido y adaptado por Leonardo Higueras (1972) y Walter Pérez actualizó los baremos en 1987. Esta Prueba fue elaborada con las técnicas del Análisis Factorial y como un cuestionario de elección en triadas (30 en total, lo que hace un conjunto de 90 ítems) permite apreciar y comparar la importancia que una persona da a los siguientes valores, actitudes o aspectos de sus relaciones con los demás: Soporte, Conformidad, Reconocimiento, Independencia, Liderazgo. La administración puede ser grupal y no hay límite de tiempo, aunque toma aproximadamente 20 minutos. La corrección se realiza a mano, mediante la aplicación de plantillas de corrección directamente sobre las hojas de respuestas. La puntuación varia entre 2, 1 o 0 puntos. La prueba se puede tomar a jóvenes a partir de los 14 años.

2) Cuestionario de Estilos Atribucionales, original de Alonso y Sánchez (1992), adaptación de Matalinares, Tueros, Yaringaño (2009) dirigido a alumnos de 11 a 15 años, de aplicación Colectiva e individual, con el propósito de evaluar los patrones atribucionales en el ámbito de las relaciones interpersonales y de los logros académicos.

Esta prueba está constituida por las siguientes áreas: 
Rosa Tueros C., María Matalinares, C. y Ornella Raymundo V.

\section{LOGROS ACADÉMICOS}

1.1 Externalización e incontrolabilidad de los resultados académicos. Hacen referencia a la atribución de los éxitos académicos a causas externas al propio sujeto no controlables por el mismo, a saber, la suerte y otras personas.

1.2 Atribución del fracaso académico a la falta de esfuerzo. Hace referencia al fracaso académico, resultado que se atribuye a la falta de esfuerzo; causa normalmente percibida como interna, variable y controlable.

1.3 Atribución del éxito académico a la habilidad. En estos elementos el éxito se atribuye a causas internas, estables y controlables.

1.4 Atribución del fracaso al profesor. El contenido de los elementos hace referencia a la atribución del fracaso al profesor. Al igual que en otros factores, incluye todos los elementos generales y específicos que se esperaba que estuviesen relacionados. Este factor se percibe como causa normalmente percibida como externa y no controlable.

1.5 Atribución del éxito al esfuerzo, exteriorización e incontrolabilidad del fracaso académico por su atribución a la mala suerte. Causa habitualmente percibida como interna, variable y controlable.

1.6 Atribución del fracaso a la falta de habilidad. El fracaso académico se atribuye a la falta de habilidad. Causa normalmente percibida como interna, estable y no controlable.

\section{RELACIONES INTERPERSONALES}

2.1 Internalización del fracaso en las relaciones interpersonales. Hacen referencia a la atribución fracaso en las relaciones interpersonales a causas internas-falta de esfuerzo o de habilidad.

2.2 Atribución del éxito en las relaciones interpersonales al esfuerzo. Hacen referencia a la atribución del éxito en las relaciones interpersonales al esfuerzo, este es el nombre que hemos dado a este factor.

2.3 Externalización del éxito en las relaciones interpersonales. En estos elementos el fracaso se atribuye a la falta de habilidad y el éxito se atribuye a la misma causa o a causas externas como la suerte o los otros (resto de los elementos).

2.3 Atribución del éxito en las relaciones interpersonales a la habilidad. El contenido de los elementos hace referencia a la atribución del éxito en las relaciones interpersonales a la habilidad y a las otras personas. Refleja la tendencia a internalizar del éxito, que se atribuye a la habilidad.

2.4 Externalización del fracaso. El contenido de los mismos hace referencia a la atribución del fracaso a la mala suerte o a acción de otras personas, esto es, a causas externas. 
Validez de contenido. Se estableció a través del criterio de 10 jueces expertos que contaban con el grado de magíster o doctor con reconocida trayectoria y experiencia en el campo profesional, para lo cual se utilizó el Coeficiente V. De Aiken. De acuerdo con Escurra (1988), en un grupo de ocho jueces se requiere que por lo menos siete estén en concordancia para que el ítem sea válido, alcanzando coeficiente $\mathrm{V}$ iguales o superiores a 0,88 , a un nivel de significación estadística de $\mathrm{p}<0,05$. A continuación se presentan los resultados obtenidos, en primer lugar en cada uno de los factores personales y luego a nivel general.

Confiabilidad. Se realizó mediante un estudio de las correlaciones entre los factores correspondientes al área de los logros académicos y al área de relaciones interpersonales. En la Tabla 2 se presentan los resultados del análisis de los componentes de los estilos atribucionales, con la media y la correlación ítem-test corregida, asumiéndose los puntajes de cada sub-escala como si fuera un ítem. Asimismo el coeficiente el coeficiente alpha de Cronbach es 0,711 como medida de confiabilidad por consistencia interna de los subtest y el área, respectivamente.

\section{RESULTADOS}

A continuación se presentan los estadísticos descriptivos e inferenciales obtenidos en el análisis de los datos obtenidos.

Tabla 1. Resultados Descriptivo en el cuestionario de Estilos Atribucionales Área de logros académicos

\begin{tabular}{|c|c|c|c|c|c|c|c|}
\hline & Escala 1 & Escala 2 & Escala 3 & Escala 4 & Escala 5 & Escala 6 & Escala 7 \\
\hline & $\begin{array}{l}\text { Externa- } \\
\text { lización e } \\
\text { incontrolabi- } \\
\text { lidad de los } \\
\text { resultados } \\
\text { académicos }\end{array}$ & $\begin{array}{c}\text { Atribución } \\
\text { del fracaso } \\
\text { académi- } \\
\text { co a la } \\
\text { falta de } \\
\text { esfuerzo }\end{array}$ & $\begin{array}{l}\text { Atribución } \\
\text { del éxito } \\
\text { académico } \\
\text { a la habi- } \\
\text { lidad }\end{array}$ & $\begin{array}{l}\text { Atribución } \\
\text { del fracaso } \\
\text { al profesor }\end{array}$ & $\begin{array}{l}\text { Atribución } \\
\text { del éxito al } \\
\text { esfuerzo }\end{array}$ & $\begin{array}{l}\text { Externaliza- } \\
\text { ción e incon- } \\
\text { trolabilidad } \\
\text { del fracaso } \\
\text { académico por } \\
\text { su atribución a } \\
\text { la mala suerte }\end{array}$ & $\begin{array}{c}\text { Atribución } \\
\text { del fracaso } \\
\text { a la falta } \\
\text { de habili- } \\
\text { dad }\end{array}$ \\
\hline Media & 20,05 & 15,53 & 14,50 & 5,76 & 11,03 & 4,77 & 5,53 \\
\hline Mediana & 21,00 & 16,00 & 15,00 & 5,00 & 11,00 & 4,00 & 5,00 \\
\hline Moda & 24 & 20 & 16 & 4 & 8 & 4 & 4 \\
\hline Desv. típ. & 4,068 & 3,624 & 4,506 & 4,356 & 5,295 & 3,401 & 3,385 \\
\hline Varianza & 16,550 & 13,135 & 20,300 & 18,973 & 28,036 & 11,565 & 11,455 \\
\hline Asimetría & $-1,597$ &,- 792 &,- 352 & ,992 & ,466 & ,873 & ,241 \\
\hline Curtosis & 3,313 & ,293 &,- 351 & 1,314 &, 524 & ,875 &,- 390 \\
\hline Rango & 24 & 17 & 22 & 24 & 30 & 16 & 16 \\
\hline Mínimo & 0 & 3 & 2 & 0 & 0 & 0 & 0 \\
\hline Máximo & 24 & 20 & 24 & 24 & 30 & 16 & 16 \\
\hline
\end{tabular}

$\mathrm{N}=400$ 
En la Tabla 1 se observa que las medias de las escalas del cuestionario de Estilos Atribucionales en el Área de logros académicos varía entre 5,00 y 21,00, en todos los casos la media recortada es similar a media, por lo que podemos afirmar que los valores extremos no modifican los valores de las medidas de tendencia central. La varianza más altas se encuentra en las escalas 5, 3 y 4 en orden descendiente. Existe mayor homogeneidad en las escalas 6 y 7. El puntaje mínimo es de 0 puntos y el puntaje máximo 24. Los rangos más altos se encuentran en las escalas 5 , 1 y 4 . La asimetría es menor a cero en el caso de las escalas 1,2 y 3; es mayor a 0 en el caso de las escalas 4, 5, 6 y 7; siendo cercanas a cero en las escalas 3 , 5 y 7. La curtosis es mayor a cero en todos los casos a excepción de las escalas 3 y 7; de acuerdo con lo anterior se afirma que en general las escalas presentan distribución asimétrica, y apuntalamiento mesocúrtico, lo que caracteriza una distribución no semejante a la normal.

Tabla 2. Resultados Descriptivos en el cuestionario de Estilos Atribucionales Área de relaciones interpersonales

\begin{tabular}{|c|c|c|c|c|c|}
\hline & Escala 8 & Escala 9 & Escala 10 & Escala 11 & Escala 12 \\
\hline & $\begin{array}{c}\text { Internalización } \\
\text { del fracaso en } \\
\text { las relaciones } \\
\text { interperso- } \\
\text { nales }\end{array}$ & $\begin{array}{l}\text { Atribución del } \\
\text { éxito en las } \\
\text { relaciones in- } \\
\text { terpersonales } \\
\text { al esfuerzo }\end{array}$ & $\begin{array}{c}\text { Externaliza- } \\
\text { ción del éxito } \\
\text { en las relacio- } \\
\text { nes interperso- } \\
\text { nales }\end{array}$ & $\begin{array}{l}\text { Atribución del } \\
\text { éxito en las } \\
\text { relaciones in- } \\
\text { terpersonales } \\
\text { a la habilidad }\end{array}$ & $\begin{array}{l}\text { Externali- } \\
\text { zación del } \\
\text { fracaso }\end{array}$ \\
\hline Media & 13,08 & 16,97 & 8,80 & 13,76 & 12,62 \\
\hline Mediana & 13,00 & 18,00 & 9,00 & 14,00 & 12,00 \\
\hline Moda & 10 & 16 & 9 & 16 & 11 \\
\hline Desv. típ. & 5,359 & 4,548 & 5,009 & 3,760 & 4,889 \\
\hline Varianza & 28,719 & 20,687 & 25,089 & 14,137 & 23,900 \\
\hline Asimetría &, 175 &,- 470 &, 252 &,- 405 &, 155 \\
\hline Curtosis &,- 418 &,- 419 &,- 556 &,- 284 & ,089 \\
\hline Rango & 28 & 19 & 22 & 16 & 25 \\
\hline Mínimo & 0 & 5 & 0 & 4 & 0 \\
\hline Máximo & 28 & 24 & 22 & 20 & 25 \\
\hline
\end{tabular}

$\mathrm{N}=400$

En la Tabla 2 se observa que las medias de las escalas del cuestionario de Estilos Atribucionales en el Área de Relaciones Interpersonales varía entre 8,80 y 16,97, en todos los casos la media recortada es similar a media, por lo que podemos afirmar 
que los valores extremos no modifican los valores de las medidas de tendencia central. La varianza más altas se encuentra en las escalas 8, 10 y 12 en orden descendiente. Existe mayor homogeneidad en la escala 11. El puntaje mínimo es de 0 puntos y el puntaje máximo 28. Los rangos más altos se encuentran en las escalas 8 y 12. La asimetría es menor a cero en el caso de las escalas 9 y 11; y es mayor a 0 en el caso de las escalas 8,10 y 12; siendo cercanas a cero en las escalas 11 y 12. La curtosis es menor a cero en todos los casos a excepción de la escala 12; de acuerdo con lo anterior se puede afirmar que en general las escalas presentan distribución asimétrica, y apuntalamiento platicúrtico, lo que caracteriza una distribución no semejante a la normal.

Tabla 3. Resultados Descriptivos en valores interpersonales

\begin{tabular}{ccccccc}
\hline & Soporte & $\begin{array}{c}\text { Confor- } \\
\text { midad }\end{array}$ & $\begin{array}{c}\text { Reconoci- } \\
\text { miento }\end{array}$ & $\begin{array}{c}\text { Indepen- } \\
\text { dencia }\end{array}$ & $\begin{array}{c}\text { Benevo- } \\
\text { lencia }\end{array}$ & Liderazgo \\
\hline $\begin{array}{c}\text { Media } \\
\text { Error típico } \\
\text { media }\end{array}$ & 15,49 & 16,01 & 10,71 & 15,04 & 18,49 & 13,06 \\
Media recortada & 0,237 & 0,321 & 0,213 & 0,393 & 0,365 & 0,299 \\
al 5\% & 15,37 & 15,91 & 10,58 & 15,15 & 18,58 & 12,89 \\
Mediana & 16,00 & 15,00 & 11,00 & 16,00 & 18,00 & 13,00 \\
Moda & 16 & 14 & 11 & 12 & 12 & 14 \\
Desv. típ. & 3,715 & 5,017 & 3,335 & 6,149 & 5,721 & 4,678 \\
Varianza & 13,800 & 25,172 & 11,123 & 37,814 & 32,726 & 21,882 \\
Asimetría &, 298 &, 353 &, 581 &,- 388 &,- 023 &, 551 \\
Curtosis &, 152 &,- 597 &, 087 &, 129 & $-1,126$ &, 800 \\
Rango & 20 & 23 & 19 & 29 & 22 & 25 \\
Mínimo & 6 & 5 & 3 & 0 & 7 & 2 \\
Máximo & 26 & 28 & 22 & 29 & 29 & 27 \\
\hline
\end{tabular}

$\mathrm{N}=400$

En la Tabla 3 se observa que las medias de las escalas valores interpersonales varía entre 18,49 y 10,71, en todos los casos la media recortada es similar a media, por lo que podemos afirmar que los valores extremos no modifican los valores de las medidas de tendencia central. La varianza más alta se encuentran en independencia y benevolencia en orden descendiente. Existe mayor homogeneidad en Reconocimiento. El puntaje mínimo es de 0 puntos y el puntaje máximo 29. Los rangos más altos se encuentran en Independencia y Liderazgo. La asimetría es menor a cero en el caso de independencia y benevolencia; y es mayor a 0 en el resto; siendo cercanas a cero en independencia y benevolencia. La curtosis es 
menor a cero solo en los casos de conformidad y benevolencia; de acuerdo con lo anterior se puede afirmar que en general las escalas presentan distribución asimétrica, y apuntalamiento leptocúrtico, lo que caracteriza una distribución no semejante a la normal.

Tabla 4. Resultados comparativos en el cuestionario de Estilos Atribucionales Área de logros académicos según sexo

\begin{tabular}{|c|c|c|c|c|c|c|c|c|}
\hline & \multirow{2}{*}{\multicolumn{2}{|c|}{$\begin{array}{c}\text { Escala } 1 \\
\text { Externalización e } \\
\text { incontrolabilidad } \\
\text { de los resultados } \\
\text { académicos }\end{array}$}} & \multicolumn{2}{|c|}{ Escala 2} & \multicolumn{2}{|c|}{ Escala 3} & \multicolumn{2}{|c|}{ Escala 4} \\
\hline & & & \multicolumn{2}{|c|}{$\begin{array}{l}\text { Atribución del } \\
\text { fracaso académi- } \\
\text { co a la falta de } \\
\text { esfuerzo }\end{array}$} & \multicolumn{2}{|c|}{$\begin{array}{c}\text { Atribución del } \\
\text { éxito académico a } \\
\text { la habilidad }\end{array}$} & \multicolumn{2}{|c|}{$\begin{array}{l}\text { Atribución del } \\
\text { fracaso al pro- } \\
\text { fesor }\end{array}$} \\
\hline & $\mathrm{H}$ & M & $\mathrm{H}$ & M & $\mathrm{H}$ & M & $\mathrm{H}$ & M \\
\hline $\mathrm{N}$ & 165 & 235 & 165 & 235 & 165 & 235 & 165 & 235 \\
\hline Media & 20,29 & 19,89 & 15,13 & 15,82 & 14,19 & 14,72 & 4,08 & 6,93 \\
\hline Mediana & 21,00 & 21,00 & 16,00 & 16,00 & 15,00 & 15,50 & 3,00 & 6,50 \\
\hline Desv. Estándar & 3,827 & 4,235 & 3,802 & 3,480 & 3,944 & 4,863 & 4,265 & 4,034 \\
\hline \multirow[t]{4}{*}{ Varianza } & 14,647 & 17,932 & 14,453 & 12,107 & 15,554 & 23,646 & 18,194 & 16,275 \\
\hline & \multicolumn{2}{|c|}{ Escala 5} & \multicolumn{2}{|c|}{ Escala 6} & \multicolumn{2}{|c|}{ Escala 7} & & \\
\hline & \multicolumn{2}{|c|}{$\begin{array}{l}\text { Atribución del } \\
\text { éxito al esfuerzo }\end{array}$} & \multicolumn{2}{|c|}{$\begin{array}{l}\text { Externalización } \\
\text { e incontrolabili- } \\
\text { dad del fracaso } \\
\text { académico por } \\
\text { su atribución a la } \\
\text { mala suerte }\end{array}$} & \multicolumn{2}{|c|}{$\begin{array}{l}\text { Atribución del } \\
\text { fracaso a la falta } \\
\text { de habilidad }\end{array}$} & & \\
\hline & $\mathrm{H}$ & M & $\mathrm{H}$ & M & $\mathrm{H}$ & M & & \\
\hline $\mathrm{N}$ & 165 & 235 & 165 & 235 & 165 & 235 & & \\
\hline Media & 7,97 & 13,17 & 4,46 & 4,99 & 4,85 & 6,00 & & \\
\hline Mediana & 8,00 & 13,00 & 4,00 & 4,00 & 5,00 & 6,00 & & \\
\hline Desv. Estándar & 3,976 & 5,054 & 3,045 & 3,624 & 3,122 & 3,490 & & \\
\hline Varianza & 15,809 & 25,543 & 9,270 & 13,133 & 9,748 & 12,182 & & \\
\hline
\end{tabular}

De acuerdo con la Tabla 4, en lo relacionado a Estilos Atribucionales Área de logros académicos; se observa que los hombres obtienen mayor promedio en la Escala 1 (Externalización e incontrolabilidad de los resultados académicos y fundamentalmente el éxito), en el resto de dimensiones de los estilos atribucionales en el área de logros académicos la mujeres obtiene mayores promedios. 
Tabla 5. Resultados comparativos en el cuestionario de Estilos Atribucionales Área de relaciones interpersonales según sexo

\begin{tabular}{|c|c|c|c|c|c|c|}
\hline & \multirow{2}{*}{\multicolumn{2}{|c|}{$\begin{array}{c}\text { Escala } 8 \\
\text { Internalización del fra- } \\
\text { caso en las relaciones } \\
\text { interpersonales }\end{array}$}} & \multirow{2}{*}{\multicolumn{2}{|c|}{$\begin{array}{c}\text { Escala } 9 \\
\text { Atribución del éxito en } \\
\text { las relaciones interper- } \\
\text { sonales al esfuerzo }\end{array}$}} & \multirow{2}{*}{\multicolumn{2}{|c|}{$\begin{array}{c}\text { Escala } 10 \\
\text { Externalización del } \\
\text { éxito en las relaciones } \\
\text { interpersonales }\end{array}$}} \\
\hline & & & & & & \\
\hline & $\mathrm{H}$ & M & $\mathrm{H}$ & M & $\mathrm{H}$ & M \\
\hline $\mathrm{N} \cdot{ }^{\circ}$ & 165 & 235 & 165 & 235 & 165 & 235 \\
\hline Media & 12,74 & 13,31 & 14,38 & 18,78 & 5,90 & 10,83 \\
\hline Mediana & 12,00 & 13,00 & 14,00 & 19,00 & 5,00 & 10,00 \\
\hline Desv. estándar & 5,149 & 5,507 & 4,684 & 3,448 & 4,232 & 4,496 \\
\hline \multirow[t]{4}{*}{ Varianza } & 26,513 & 30,328 & 21,937 & 11,890 & 17,910 & 20,214 \\
\hline & \multicolumn{2}{|c|}{ Escala 11} & \multicolumn{2}{|c|}{ Escala 12} & & \\
\hline & \multicolumn{2}{|c|}{$\begin{array}{l}\text { Atribución del éxito en } \\
\text { las relaciones interper- } \\
\text { sonales a la habilidad }\end{array}$} & \multicolumn{2}{|c|}{$\begin{array}{l}\text { Externalización del } \\
\text { fracaso }\end{array}$} & & \\
\hline & $\mathrm{H}$ & M & $\mathrm{H}$ & M & & \\
\hline $\mathrm{N}$ & 165 & 235 & 165 & 235 & & \\
\hline Media & 11,45 & 15,38 & 13,15 & 12,25 & & \\
\hline Mediana & 12,00 & 16,00 & 13,00 & 12,00 & & \\
\hline Desv. Estándar & 3,659 & 2,889 & 5,228 & 4,619 & & \\
\hline Varianza & 13,390 & 8,348 & 27,328 & 21,336 & & \\
\hline
\end{tabular}

En la Tabla 5, en lo relacionado a Estilos Atribucionales Área de relaciones interpersonales; se observa que los hombres obtienen mayor promedio en la Escala 12 (Externalización del fracaso), en el resto de dimensiones de los estilos atribucionales en el área de relaciones interpersonales las mujeres obtiene mayores promedios.

Tabla 6. Resultados comparativos en valores interpersonales según sexo

\begin{tabular}{ccccccc}
\hline & \multicolumn{2}{c}{ Soporte } & \multicolumn{2}{c}{ Conformidad } & \multicolumn{2}{c}{ Reconocimiento } \\
\cline { 2 - 7 } & $\mathrm{H}$ & $\mathrm{M}$ & $\mathrm{H}$ & $\mathrm{M}$ & $\mathrm{H}$ & $\mathrm{M}$ \\
\hline $\mathrm{N} .^{\circ}$ & 165 & 235 & 165 & 235 & 165 & 235 \\
Media & 14,45 & 16,22 & 13,80 & 17,56 & 9,60 & 11,49 \\
Mediana & 14,00 & 16,00 & 12,00 & 17,00 & 8,00 & 11,00 \\
Desv. estándar & 4,051 & 3,282 & 4,858 & 4,539 & 3,280 & 3,158 \\
Varianza & 16,410 & 10,772 & 23,600 & 20,598 & 10,762 & 9,972 \\
\hline
\end{tabular}


Rosa Tueros C., Maria Matalinares, C. y Ornella Raymundo V.

\begin{tabular}{ccccccc}
\hline & \multicolumn{2}{c}{ Independencia } & \multicolumn{2}{c}{ Benevolencia } & \multicolumn{2}{c}{ Liderazgo } \\
\cline { 2 - 7 } & $\mathrm{H}$ & $\mathrm{M}$ & $\mathrm{H}$ & $\mathrm{M}$ & $\mathrm{H}$ & $\mathrm{M}$ \\
\cline { 2 - 7 } $\mathrm{N}^{\circ}$ & 165 & 235 & 165 & 235 & 165 & 235 \\
\hline Media & 9,50 & 18,92 & 17,84 & 18,94 & 13,78 & 12,55 \\
Mediana & 12,00 & 18,00 & 17,00 & 19,00 & 13,00 & 12,00 \\
Desv. Estándar & 4,525 & 3,647 & 6,016 & 5,479 & 4,993 & 4,390 \\
Varianza & 20,472 & 13,302 & 36,195 & 30,025 & 24,932 & 19,270 \\
\hline
\end{tabular}

De acuerdo con la Tabla 6, en lo relacionado a valores interpersonales; se observa que los hombres obtienen mayor promedio en Liderazgo y por el contrario las mujeres obtienen mayor promedio en los valores Soporte, Conformidad, Reconocimiento, Independencia y Benevolencia.

A continuación se presentan los resultados de las correlaciones obtenidas en las variables de estudio con el propósito de contrastar las hipótesis planteadas.

Tabla 7. Test de Kolmogorov Smirnov, para comprobar una distribución normal

\begin{tabular}{lccc}
\hline & K-S & gl & Sig. \\
\hline Escala 1: Externalización e incontrolabilidad de los resultados académicos. &, 074 & 400 &, 000 \\
Escala 2: Atribución del fracaso académico a la falta de esfuerzo. &, 128 & 400 &, 000 \\
Escala 3: Atribución del éxito académico a la habilidad. &, 095 & 400 &, 000 \\
Escala 4: Atribución del fracaso al profesor. &, 124 & 400 &, 000 \\
Escala 5: Atribución del éxito al esfuerzo. &, 149 & 400 &, 000 \\
Escala 6: Externalización e incontrolabilidad del fracaso académico por su &, 134 & 400 &, 000 \\
atribución a la mala suerte. &, 105 & 400 &, 000 \\
Escala 7: Atribución del fracaso a la falta de habilidad. &, 055 & 400 &, 020 \\
Escala 8: Internalización del fracaso en las relaciones interpersonales. &, 095 & 400 &, 000 \\
Escala 9: Atribución del éxito en las relaciones interpersonales al esfuerzo. &, 089 & 400 &, 000 \\
Escala 10: Externalización del éxito en las relaciones interpersonales. &, 099 & 400 &, 000 \\
Escala 11: Atribución del éxito en las relaciones interpersonales a la habilidad. &, 068 & 400 &, 001 \\
Escala 12: Externalización del fracaso. &, 106 & 400 &, 000 \\
Soporte &, 141 & 400 &, 000 \\
Conformidad &, 119 & 400 &, 000 \\
Reconocimiento &, 107 & 400 &, 000 \\
Independencia &, 100 & 400 &, 000 \\
Benevolencia &, 081 & 400 &, 000 \\
Liderazgo & &
\end{tabular}


De acuerdo con la Tabla 7, se observa el estadístico de Kolmogorov Smirnov en todos los casos presenta un nivel significatividad de 0,00 . Puesto que el valor del nivel crítico es menor que 0,05 , en todos los casos, rechazamos la hipótesis de normalidad y concluimos que las puntuaciones de las áreas del aprendizaje experiencial no se ajustan a una distribución normal. De este modo se decide el uso de estadísticos no paramétricos.

Tabla 8. Coeficientes de Correlación de Spearman en Estilos Atribucionales del área logros académicos y valores interpersonales

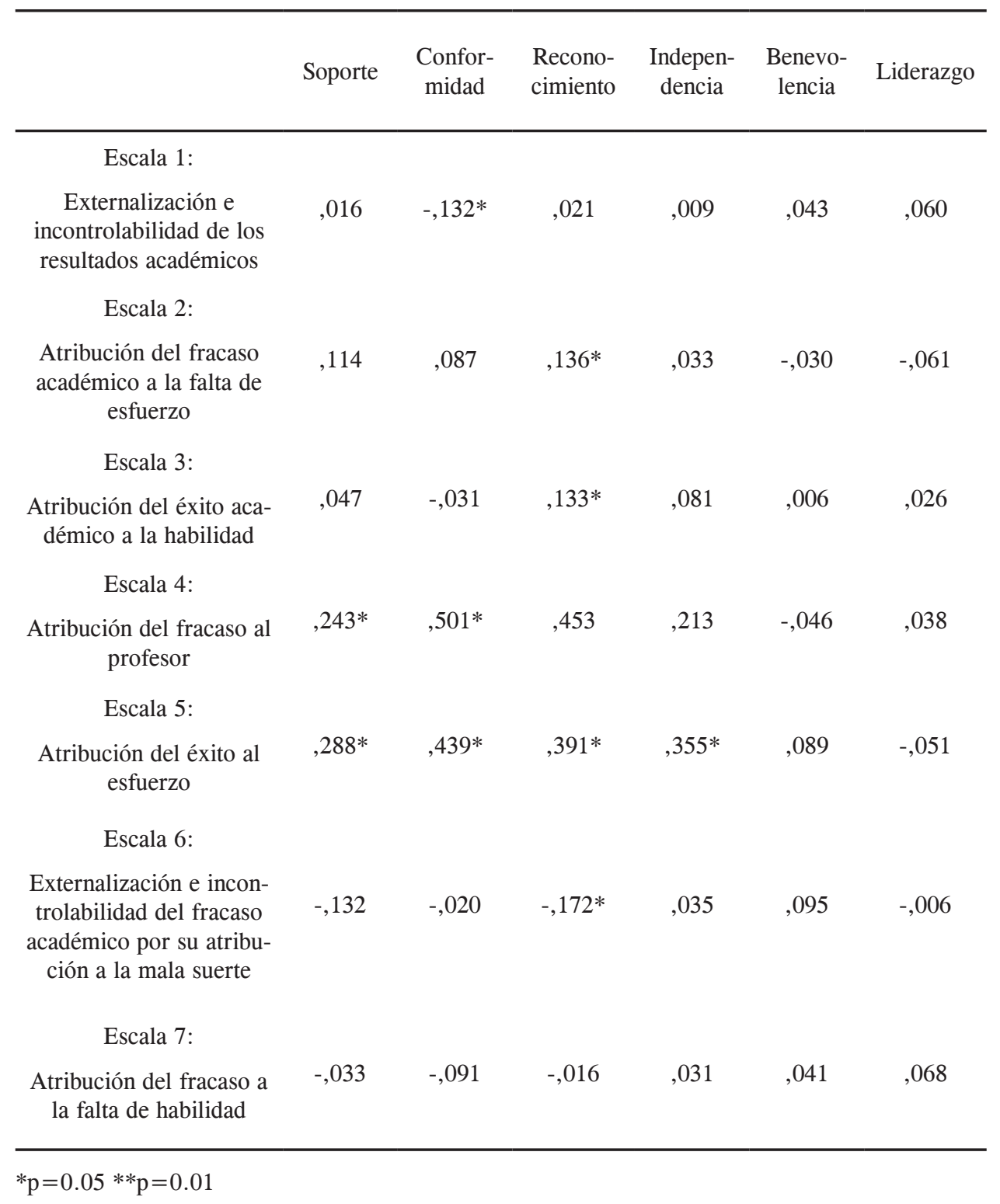


Basándose en los resultados de la Tabla 8, se puede afirmar que en el caso de soporte se encuentran correlaciones positivas con atribución del fracaso al profesor y atribución del éxito al esfuerzo; en el caso de conformidad se encuentran correlaciones positivas con externalización e incontrolabilidad de los resultados académicos, atribución del fracaso al profesor y atribución del éxito al esfuerzo; en reconocimiento se encuentran correlaciones positivas con atribución del fracaso académico a la falta de esfuerzo, atribución del éxito académico a la habilidad y atribución del éxito al esfuerzo, y correlaciones negativa con externalización e incontrolabilidad del fracaso académico por su atribución a la mala suerte. Finalmente se encuentra correlación positiva entre Independencia y Atribución del éxito al esfuerzo.

Tabla 9. Coeficientes de Correlación de Spearman en Estilos Atribucionales del área relaciones interpersonales y los componentes de los valores interpersonales

\begin{tabular}{|c|c|c|c|c|c|c|}
\hline & Soporte & $\begin{array}{l}\text { Confor- } \\
\text { midad }\end{array}$ & $\begin{array}{l}\text { Recono- } \\
\text { cimiento }\end{array}$ & $\begin{array}{l}\text { Indepen- } \\
\text { dencia }\end{array}$ & $\begin{array}{l}\text { Benevo- } \\
\text { lencia }\end{array}$ & Liderazgo \\
\hline \multicolumn{7}{|l|}{ Escala 8: } \\
\hline $\begin{array}{l}\text { Internalización del fra- } \\
\text { caso en las relaciones } \\
\text { interpersonales }\end{array}$ &,- 033 &,- 091 &,- 016 & ,031 &, 041 & ,068 \\
\hline \multicolumn{7}{|l|}{ Escala 9: } \\
\hline $\begin{array}{l}\text { Atribución del éxito en } \\
\text { las relaciones interper- } \\
\text { sonales al esfuerzo }\end{array}$ &, $279 * *$ &, $479 * *$ & $425 * *$ & ,370** & ,012 & ,066 \\
\hline \multicolumn{7}{|l|}{ Escala 10: } \\
\hline $\begin{array}{l}\text { Externalización del } \\
\text { éxito en las relaciones } \\
\text { interpersonales }\end{array}$ &, $259 * *$ &, $406 * *$ &, $419 * *$ &, $371 * *$ & ,049 & ,010 \\
\hline \multicolumn{7}{|l|}{ Escala 11: } \\
\hline $\begin{array}{l}\text { Atribución del éxito en } \\
\text { las relaciones interper- } \\
\text { sonales a la habilidad }\end{array}$ &, $413 * *$ &, $458 * *$ &, $446 * *$ &, $357 * *$ &,- 049 &,- 048 \\
\hline \multicolumn{7}{|l|}{ Escala 12: } \\
\hline $\begin{array}{l}\text { Externalización del } \\
\text { fracaso }\end{array}$ &,$- 156^{*}$ &,- 051 &,$- 137 *$ &,- 118 & ,006 &, 017 \\
\hline
\end{tabular}


De acuerdo con los resultados de la Tabla 9, se establece que en el caso del valor interpersonal soporte se encuentran correlaciones positivas con atribución del éxito en las relaciones interpersonales al esfuerzo, atribución del éxito en las relaciones y externalización del éxito en las relaciones interpersonales, por el contrario correlación negativa con externalización del fracaso; en el caso de conformidad se encuentran correlaciones positivas con atribución del éxito en las relaciones interpersonales al esfuerzo, externalización del éxito en las relaciones interpersonales y atribución del éxito en las relaciones interpersonales a la habilidad; en reconocimiento se encuentran correlaciones positivas con atribución del éxito en las relaciones interpersonales al esfuerzo, externalización del éxito en las relaciones interpersonales y atribución del éxito en las relaciones interpersonales a la habilidad, y correlación negativa con externalización del fracaso, se encuentra correlación positiva entre Independencia y atribución del éxito en las relaciones interpersonales al esfuerzo, externalización del éxito en las relaciones interpersonales y atribución del éxito en las relaciones interpersonales a la habilidad. Finalmente se halló correlación negativa en la externalización del fracaso con los componentes soporte y reconocimiento de los valores interpersonales.

Tabla 10. Prueba sobre diferencia de medias de Mann-Whitney, en Estilos Atribucionales área logros académicos según sexo

\begin{tabular}{|c|c|c|c|c|c|c|c|}
\hline & Escala 1 & Escala 2 & Escala 3 & Escala 4 & Escala 5 & Escala 6 & Escala 7 \\
\hline & $\begin{array}{l}\text { Externali- } \\
\text { zación e in- } \\
\text { controlabi- } \\
\text { lidad de los } \\
\text { resultados } \\
\text { académicos }\end{array}$ & $\begin{array}{c}\text { Atribución } \\
\text { del fracaso } \\
\text { académi- } \\
\text { co a la } \\
\text { falta de } \\
\text { esfuerzo }\end{array}$ & $\begin{array}{l}\text { Atribución } \\
\text { del éxito } \\
\text { académico } \\
\text { a la habili- } \\
\text { dad }\end{array}$ & $\begin{array}{l}\text { Atribución } \\
\text { del fracaso } \\
\text { al profesor }\end{array}$ & $\begin{array}{l}\text { Atribución } \\
\text { del éxito } \\
\text { al esfuerzo }\end{array}$ & $\begin{array}{l}\text { Externaliza- } \\
\text { ción e incon- } \\
\text { trolabilidad } \\
\text { del fracaso } \\
\text { académico por } \\
\text { su atribución a } \\
\text { la mala suerte }\end{array}$ & $\begin{array}{l}\text { Atribu- } \\
\text { ción del } \\
\text { fracaso a } \\
\text { la falta de } \\
\text { habilidad }\end{array}$ \\
\hline $\mathrm{U}$ & 6912,500 & 6603,500 & 6711,000 & 3948,000 & 2879,500 & 6896,500 & 5899,500 \\
\hline $\mathrm{Z}$ &,- 664 & $-1,232$ & $-1,031$ & $-6,108$ & $-8,061$ &,- 692 & $-2,525$ \\
\hline Sig. &, 507 & ,218 & ,303 &, 000 &, 000 & ,489 & ,012 \\
\hline
\end{tabular}

Variable de agrupación: sexo

La Tabla 10 ofrece el estadístico U de Mann-Whitney, la tipificación Z y la Significación asintótica bilateral (sig.). En relación a la significación, esta asume valores menores a 0,05 en los casos de la Escala 4 (Atribución del fracaso al profesor), la Escala 5 (Atribución del éxito al esfuerzo) y la Escala 7 (Atribución del fracaso a la falta de habilidad), por tanto, se rechaza la hipótesis de igualdad de promedios y se concluye que los grupos definidos por la variable sexo proceden de poblaciones con distinto promedio en las escalas mencionadas. 
Tabla 11. Prueba sobre diferencia de medias de Mann-Whitney, en Estilos Atribucionales área de relaciones interpersonales según sexo

\begin{tabular}{rccccc}
\hline & Escala 8 & Escala 9 & Escala 10 & Escala 11 & Escala 12 \\
$\begin{array}{c}\text { Internalización } \\
\text { del fracaso en } \\
\text { las relaciones } \\
\text { interpersonales }\end{array}$ & $\begin{array}{c}\text { Atribución del } \\
\text { éxito en las } \\
\text { relaciones in- } \\
\text { terpersonales } \\
\text { al esfuerzo }\end{array}$ & $\begin{array}{c}\text { Externaliza- } \\
\text { ción del éxito } \\
\text { en las relacio- } \\
\text { nes interperso- } \\
\text { nales }\end{array}$ & $\begin{array}{c}\text { Atribución del } \\
\text { éxito en las } \\
\text { relaciones in- } \\
\text { terpersonales a } \\
\text { la habilidad }\end{array}$ & $\begin{array}{c}\text { Externali- } \\
\text { zación del } \\
\text { fracaso }\end{array}$ & \\
\hline $\mathrm{U}$ & 6834,000 & 3277,500 & 2797,000 & 2836,500 & 6406,500 \\
$\mathrm{Z}$ &,- 804 & $-7,334$ & $-8,216$ & $-8,156$ & $-1,589$ \\
Sig. &, 422 &, 000 &, 000 &, 000 &, 112 \\
\hline
\end{tabular}

Variable de agrupación: sexo

La Tabla 11 ofrece el estadístico U de Mann-Whitney, la tipificación Z y la Significación asintótica bilateral (sig.). En relación a la significación esta asume valores menores a 0,05 en los casos de la Escala 9 (atribución del éxito en las relaciones interpersonales al esfuerzo), Escala 10 (externalización del éxito en las relaciones interpersonales) y la Escala 11 (atribución del éxito en las relaciones interpersonales a la habilidad), por tanto, se rechaza la hipótesis de igualdad de promedios y se concluye que los grupos definidos por la variable sexo proceden de poblaciones con distinto promedio en las escalas mencionadas.

Tabla 12. Prueba sobre diferencia de medias de Mann-Whitney, en valores interpersonales según sexo

\begin{tabular}{rcccccc}
\hline & Soporte & Conformidad & $\begin{array}{c}\text { Reconoci- } \\
\text { miento }\end{array}$ & Independencia & Benevolencia & Liderazgo \\
\hline $\mathrm{U}$ & 5108,500 & 3799,500 & 4736,500 & 353,500 & 6456,000 & 6370,000 \\
$\mathrm{Z}$ & $-3,983$ & $-6,378$ & $-4,672$ & $-12,711$ & $-1,499$ & $-1,658$ \\
Sig. &, 000 &, 000 &, 000 &, 000 &, 134 &, 097 \\
\hline
\end{tabular}

Variable de agrupación: sexo

La Tabla 12 ofrece el estadístico U de Mann-Whitney, la tipificación Z y la Significación asintótica bilateral (sig.). En relación a la significación esta asume valores menores a 0,05 en los casos de la Soporte, Conformidad, Reconocimiento, e Independencia, por tanto, se rechaza la hipótesis de igualdad de promedios y se concluye que los grupos definidos por la variable sexo proceden de poblaciones con distinto promedio en las escalas mencionadas. 


\section{DISCUSIÓN DE RESULTADOS}

La presente investigación demostró que existe relación entre los valores interpersonales y los estilos atributivos de estudiantes del 5. ${ }^{\text {to }}$ año de educación secundaria.

$\mathrm{Al}$ analizar más detalladamente las atribuciones en torno a los logros académicos y las relaciones interpersonales. Se encontró que el caso del valor interpersonal soporte, éste se relaciona con atribuir el fracaso al profesor pero también con atribuir el éxito académico y las relaciones interpersonales al esfuerzo, además el desarrollo del valor soporte se asocia con asumir el éxito en las relaciones interpersonales a condiciones externas y de habilidad (Gordon, 2003). Esto indicaría que al ser tratado con amabilidad, comprensión y necesidad de apoyo por parte de los demás se está favoreciendo a un estilo atributivo que se sustenta en la relación con el entorno; siendo la comunicación con el docente y el grupo de pares un factor que incide en el rendimiento académico y motivación hacia el aprendizaje. Por el contrario soporte se asume como un valor contrario, al asumir el fracaso como una cuestión externa. González y Tourón (1994) refieren que para lograr patrones motivacionales adaptativos que favorezcan la motivación de rendimiento, y aumentar los juicios del estudiante para atribuir sus éxitos a la capacidad o al esfuerzo, se debe favorecer un trato asertivo y respetuoso, siendo por el contrario un ambiente que no facilite el soporte, si esto se da, puede estimular patrones desadaptativos que inhiben la motivación o disminuyen el rendimiento, lo cual se explicaría cuando el sujeto atribuye sus éxitos a factores como el azar y sus fracasos a su baja capacidad, siendo la falta de soporte algo fuera de su control y de naturaleza estable.

$\mathrm{Al}$ encontrar una relación positiva en el área de relaciones interpersonales atribución del éxito en las relaciones interpersonales al esfuerzo de los estilos atribucionales con los componentes de los valores interpersonales soporte y conformidad. Esto indica que mientras los alumnos atribuyan el éxito en la relaciones interpersonales al esfuerzo ellos perciben que reciben apoyo por parte de los demás, son tratados con amabilidad; por consiguiente ellos se esfuerzan por hacer lo socialmente correcto.

El haber hallado correlación negativa entre los componentes de soporte y reconocimiento de los valores interpersonales, con la escala externalización del fracaso de los estilos atribucionales; indica que a mayor soporte y reconocimiento entendido estos como el ser tratados con amabilidad y consideración hay una menor atribución de los alumnos al fracaso a la mala suerte o a la acción de otras personas, esto es a causas externas.

Si tenemos en cuenta que en el valor interpersonal de Reconocimiento, el cual determina la necesidad de ser admirado, reconocido, estimado, valorado e importante para los demás, se asocia con la atribución del fracaso académico a la falta de esfuerzo, pero también en atribuir el éxito académico a la habilidad y éxito al esfuerzo, asociación similar en relación a la atribución del éxito en las relaciones interpersonales al esfuerzo y habilidad, además de externalizar el éxito en las relaciones interpersonales, debido a esto se considera que la necesidad de ser estimado favorece una actitud de esfuerzo basado en la habilidad. Pero el reconocimiento social establece como determinante el ser un valor opuesto a considerar externo 
e incontrolable el fracaso académico y asociarlo a situaciones fortuitas de sentido negativo o fracaso. Resultado también señalado por Murakami (2005), quien refirió que los jóvenes que se sienten reconocidos por los demás, también valoran más la independencia, lo cual refleja un accionar más individualista.

Al considerar la búsqueda de autonomía, libertad para decidir y tomar decisiones propias se estableció relación con la atribución al éxito académico e interpersonal al esfuerzo, atribuyendo casusas externas y personales debido al logro de habilidades de comunicación o de acción. Estos indicadores son similares a los señalados por Murakami (2005) al examinar 350 estudiantes de 5 to $^{\text {te }}$ secundaria de Lima y Chulucanas, concluyendo que los estudiantes de Lima tienen una mayor tendencia a no asumir las normas impuestas debido a que se encuentran en busca de alcanzar estabilidad y seguridad en la vida, reflejando un accionar más individualista, buscando el beneficio propio, que podría ser causado por la gran competencia que existe en el ámbito de la ciudad de Lima donde cada uno tiene que velar por sí mismo y luchar constantemente por sobresalir en un entorno competitivo.

Al considerar las diferencias de género en relación a los valores interpersonales o estilos atributivos se observó que en los alumnos varones el éxito o fracaso en estudios depende de un factor externo que ellos mismos no pueden controlar, a diferencia de las mujeres en las cuales esta percepción es más estable y no hay un preferencia en los estilos atribucionales asociado a los logros académicos. Además se observa que en la atribución de éxito en el área de relaciones interpersonales; los alumnos obtienen mayor promedio en la externalización del fracaso y las mujeres atribuyen que las relaciones interpersonales están determinadas por distintas causas que no son atribuibles al contexto externo.

Al comparar los valores interpersonales se observar que los varones asumen como valor interpersonal principal el Liderazgo. Una explicación a este hallazgo podría estar en el hecho de que se han creado expectativas de la concepción de las personas y de su función en la sociedad en función de su género, así se atribuyen habilidades, capacidades, destrezas, etc., de acuerdo con el género al que pertenecen. Los estereotipos creados explicarían la diferencia de liderazgo. Estos estereotipos son tan arraigados en la sociedad peruana, que son muy difíciles de erradicar en las prácticas cotidianas y en la visión del mundo y de la realidad que nos rodea, por lo tanto también se van a evidenciar en el ambiente escolar donde el estereotipo tradicionalmente asocia al liderazgo con el género masculino (Munduate 2003). Los resultados mostraron que las mujeres dan preferencia a los valores Soporte, Conformidad, Reconocimiento, Independencia y Benevolencia. Así como señalan Weiner y Graham (1989) una atribución no es la anticipación de las emociones sino los procesos cognitivos que determinan la evaluación de la calidad del logro, se podría establecer que las alumnas asignan con mayor énfasis un locus interno en la causalidad, siendo esta estable o constante, pero que es controlable por uno mismo; aunque si bien es verdad que se afirma que las mujeres potencian el rol de las emociones en sus atribuciones, podemos considerar que en el sentido actual de las reacciones afectivas en las mujeres, está dirigido a un creciente logro académico y personal. 


\section{CONCLUSIONES}

1. Existe relación entre relación entre los valores interpersonales y los estilos atributivos.

2. Existen diferencias significativas en valores interpersonales de estudiantes del $5{ }^{\text {to }}$ grado de secundaria según la variable sexo.

3. Existe relación significativa en estilos atributivos de estudiantes del $5 .{ }^{\text {to }}$ grado de secundaria, según la variable sexo.

\section{REFERENCIAS BIBLIOGRÁFICAS}

Aguilar, S. (2002). Estudio comparativo de los valores interpersonales en los alumnos con alta y baja autoestima de los tres primeros ciclos de la escuela de Psicología de la Universidad Señor de Sipán en la ciudad de Chiclayo. Tesis de licenciatura en Psicología.

Alcalde, C.; Marchena, E. y García M. (2006). Los valores interpersonales. Análisis de una muestra de alumnos de formación profesional de un centro de recuperación de minusválidos físicos. Universidad de Cádiz. Recuperado el 28 de octubre 2009, de http://campus.usal.es/ inico/investigacion/jornadas/jornada1/posters/p6.html.

Alonso, M.; Pereira, M. y Soto, J. (2003). La educación en valores a través de la música. Marco teórico y estrategias de intervención. En Benso Calvo, M y Pereira Domínguez, M. ${ }^{a}$ C. (coords.). El profesorado de Enseñanza Secundaria. Retos ante el nuevo milenio. Concello de Ourense, Fundación Santa María y Universidad de Vigo. Ourense. Edita Aurea, pp. 135-202.

Buey, F., Romero, M. (2003). Influencia de las expectativas en el rendimiento académico. Aula abierta. N. ${ }^{\circ} 81$, pp. 99-110.

Carmona, B., y León, M. (2005). Análisis correlacional entre las inteligencias múltiples interpersonales e intrapersonales y valores interpersonales, en estudiantes de I ciclo de la carrera de Psicología de la Universidad César Vallejo de la ciudad de Trujillo. Tesis de licenciatura.

Emiro, J, Martínez, G, Soto, J. Martínez, F y Baena, B. (2009). Valores personales e interpersonales en adolescentes y adultos de la ciudad de Medellín y el área metropolitana. Revista Diversitas, Perspectivas en Psicología - Vol. 5, N. ${ }^{\circ} 1$, pp. 125-139.

Escurra, L. M. (1991). Construcción de una prueba de Inteligencia Emocional. En: Revista de Investigación en Psicología, Vol. 3, N. ${ }^{\circ}$ 1. Julio. Lima: UNMSM, Facultad de Psicología, Instituto de Investigaciones Psicológicas, pp. 71- 85.

Gordon, L. V. (1977). Cuestionario de Valores Interpersonales. Madrid: TEA Ediciones.

González, M.C. y Tourón, J. (1994). Autoconcepto y rendimiento escolar. Sus implicaciones en la motivación y en la autorregulación del aprendizaje. Navarra: EUNSA.

González, R., Valle, A., Suárez, J., Fernández, P. (1999). Un modelo integrador explicativo de las relaciones entre metas académicas, estrategias de aprendizaje y rendimiento académico. Revista de investigación educativa. 17(1), pp. 47-70. 
Guerrero, M. (2005). Relación entre los acontecimientos vitales críticos y los valores interpersonales en los alumnos de 5. ${ }^{\text {to }}$ de secundario del Centro Educativo Nacional El Indoamericano, de Río seco. Tesis de licenciatura.

Herrera, M. (2007). Los valores de los adolescentes de un centro español de educación secundaria en el extranjero. Revista Iberoamericana de Educación, 42(3).

Herrera, M. y Herrera, F. (2010). ¿Tienen los adolescentes los mismos Valores que sus padres? Un estudio con Alumnado Ceutí de educación secundaria. REOP. Vol. 21, N. ${ }^{\circ}$ 2, pp. 401-411.

Matalinares, M., Tueros, R. y Yaringaño, J. (2009). Adaptación Psicométrica del cuestionario de estilos atributivos. Revista de Investigaciones psicológicas 12(1), pp. 173-189.

Matalinares, M., Sotelo, L, Sotelo, N., Arenas, C., Dioses, A., Yaringaño, J., Muratta, R., Pareja, C. (2009). Juicio Moral y valores interpersonales en estudiantes de secundaria de las ciudades de Lima y Jauja. Revista de Investigación Psicológica, 12(2), pp. 115-132.

Matalinares, M., Yaringaño, J., Sotelo, L., Sotelo, N., Arenas, C., Díaz, G., Dioses, A., Encalada, M., Tipacti, R, Bazán, M. y Huari, Y. (2010) Relación entre los estilos tribucionales y los estilos de aprendizaje en estudiantes de secundaria de Lima Metropolitana. Revista de Investigaciones Psicológicas 13(2), pp. 101-116.

Munduate, L. (2003). Género y liderazgo diferencias entre hombres y mujeres en el acceso a los puestos directivos disponible en http://personal.us.es/munduate/genero.pdf

Murakami, M. (2005). Valores interpersonales en alumnos de 5. ${ }^{\text {to }}$ año de educación secundaria en colegios estatales mixtos de Lima y Piura. Tesis de licenciado en Psicología. Lima: UNIFE.

Pardo A. y Tapia, J. (1990). Motivar en el aula. Servicio de Publicaciones. Univ. Autónoma. Madrid.

Romero, J. y Villegas, C. (2006). Análisis comparativo de los valores interpersonales entre los estudiantes del primer ciclo respecto a los de undécimo ciclo de la carrera profesional de Psicología de la Universidad Privada César Vallejo de la ciudad de Trujillo. Tesis de licenciatura en Psicología.

Tapia, J., Mateos, M. y Gutiérrez, F.(1994). Entrenamiento metacognitivo: desarrollo de programas para la mejora de la comprensión lectora y los procesos de razonamiento. Universidad Autónoma. Madrid. [Edición en microficha] ISBN: 84-7477-486-1

Valenzuela, J. (2007) Exigencia académica y atribución causal: ¿qué pasa con la atribución al esfuerzo cuando hay una baja significativa en la exigencia académica? Pontificia Universidad católica de Chile Educere Vol. 11 N. ${ }^{\circ} 37$ disponible en http://www. scielo.org.ve/scielo.php?pid $=$ S1316-49102007000200014\&script $=$ sci_arttext

Weiner, B. (1970). New conceptions in the study of achievement motivation. En Maher, B.A. (ed.). Progress in experimental personality research, V. Nueva York: Academic Press.

Weiner, B. (1974). Achievement motivation and attribution theory. Morristown, NJ: General Learning Press. 
Weiner, B. (1979). A theory of motivation for some classroom experiences. Journal of Educational Psychology, 71, 3-25.

Weiner, B. (1983). Some Troughts about Feelings. En Paris, S.G., Olson, G.M., Stevenson, H.W. (eds.), Learning and Motivation in the Classroom (pp. 165-178). Hillsdale, N.J.: Lawrence Erlbaum.

Weiner, B. (1985). An Attributional Theory of Achievement Motivation and Emotion. Psychological Review, 92 (4), 548-573.

Weiner, B. (1986). An Attributional Theory of Motivation and Emotion. New York: Springer-Verlag.

Weiner, B. y Graham, S. (1989). Understanding the Motivational Role of Affect: Lifespan research from an Attributional Perspective. Cognition and Emotion, 3, 401-419. 